\title{
Message of ASI President
}

\author{
Shiva K. Misra ${ }^{1}$
}

Received: 22 January 2017 / Accepted: 22 January 2017 / Published online: 3 February 2017

(C) Association of Surgeons of India 2017

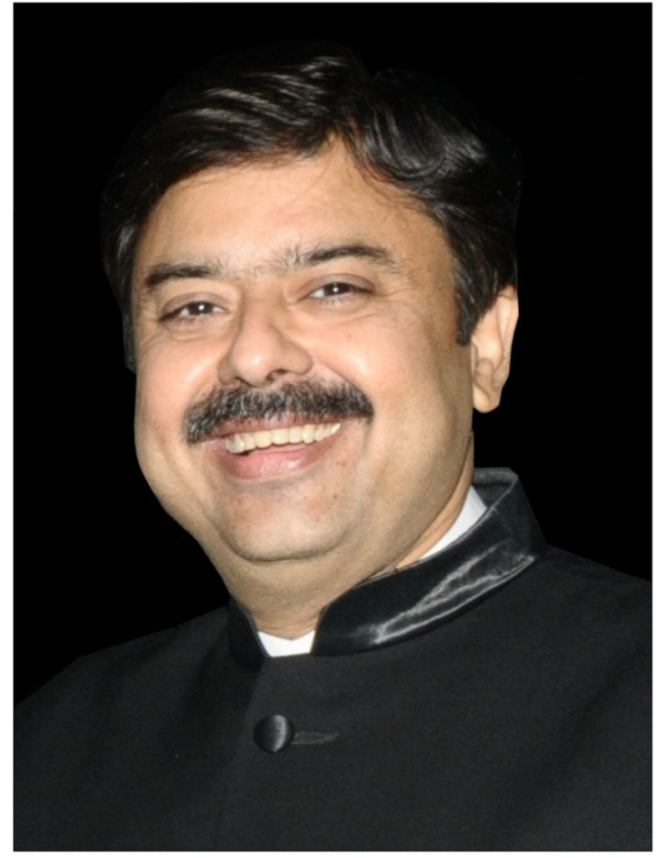

Dear Friends,

Wish you all a very happy, peaceful, and prosperous 2017.

I am thankful to all the members for electing me as the President of ASI 2017. I took charge as the 75th President of the Association of Surgeons of India at the 76th Annual Conference of the Association at Mysuru. I

Shiva K. Misra

drshivakmisra@gmail.com; http://www.drshivakmisra.com

1 Mariampur Hospital, Kanpur 208005, India promise to abide by my duties as per the ASI Constitution and fulfill all my responsibilities to strengthen ASI and take academics and other activities to newer heights as per expectation of the members.

Being a surgeon is a very tough job. It is the most physically and psychologically demanding profession in the world, but it carries many rewards too, both professionally and financially. There will be strong demands for surgeons in the next 5 to 10 years. Salaries will continue to rise. But a surgeon has to be a good team leader to be successful professionally. The difference between God and a surgeon is that nobody wants an appointment with God. The desperate demand for good surgeons has always ensured that they are among the most powerful people in the society.

"Sushruta Samhita" (Sushruta's compendium), which describes the ancient tradition of surgery in Indian medicine, is considered as one of the most brilliant gems in Indian medical literature. This treatise contains detailed descriptions of teachings and practice of the great ancient surgeon Sushruta which has considerable surgical knowledge of relevance even today. We are no stranger to the work of people such as Joseph Lister (18271912), the Englishman who brought a revolution in the practice of surgery, his advocacy of the use of carbolic acid (phenol) as an antiseptic, and was dubbed the "Father of Modern Surgery" as a result.

Ever since humans have first learned to make and handle tools, they have employed their talents to develop surgical techniques, each time more sophisticated than the last; however, until the industrial revolution, surgeons were incapable of overcoming the three principal obstacles which had plagued the medical profession from its infancy-bleeding, pain, and infection. Advances in these fields have transformed surgery from 
a risky "art" into a scientific discipline capable of treating many diseases and conditions.

Surgical postgraduates entering into general surgical practice are dwindling. Majority now pursue further fellowship training in surgical subspecialties. There prevails a lack of readiness to practice probably due to nationwide rise in the popularity of laparoscopic surgery. Laparoscopy has now become "Mainstream" in surgical training and practice. The number of residents involved in junior-level surgeries which were earlier performed conventionally and are now performed more preferably by laparoscopy has actually gone down. This is not compensated for by an increase in residents' involvement in open surgeries. Residents are spending more time on the rounds of patients on the floor and less time operating. Straightforward and thus appropriate "junior-level" cases (appendectomies, cholecystectomies) now require advanced laparoscopic skills. Thus, the concept of "Transition to Practice Fellowships," which provides new surgeons with further training in general surgical skills and procedures, is required for a private practice. Along with the abovementioned also included are supplementary training methods outside of the operating room including surgical simulation experiences. The graduating surgical residents continue to report unease and dissatisfaction with their own operating skills, and the need for timely intervention is obvious.

There has been tremendous advancement in surgery but safer surgery or patient safety in surgery is my prime concern. Identifying good skill centers across the country and establishing state of art skill center at Chennai Headquarters for the benefit of our members is my top priority. Falling ethical standards in surgical practice also need to be well addressed, and we will come out with guidelines for the ethical surgical practice. Work on various surgical protocols has already begun. Five to six redesigned zonal CMEs have been planned across the country by the Headquarters for the benefit of postgraduates and young surgeons.

In addition to the above ASI is also doing many academic activities along with state chapters and specialty sections round the year so as to help our colleagues to upgrade their knowledge and sharpen their skills as well. ASI would be identifying high volume centers in various specialties across the country for short-term and long-term training programs for its inquisitive members. Similarly, few highly specialized and advanced centers are being identified where special procedures such as liver transplant, high-tech minimal invasive surgery, specialized trauma management, oncology services in different organs, and specialized GI surgical procedures are performed, and surgeons from India and other countries will have the privilege of proper training. This global outlook is necessary as India is a rapidly advancing nation with centers of excellence at par with many international institutions. The SAARC and ASEAN countries with India have formed a group of learning and teaching with each other's cooperation so as to share their knowledge on a common platform. It will be a formidable group of surgical learning in the future.

ASI is a vibrant organization with state chapters and specialty sections always putting up social and public awareness programs. Each state must organize at least one mega surgical camp for common surgical problems and perform free operations wherever required. Every surgeon of this country is doing a lot of surgical procedures for free but is going unnoticed. As a part of our obligation and duty to mankind and also social responsibility, I urge you to at least perform one operation a year free of cost and report it to the ASI office with details to inspire your colleagues and future generation surgeons.

Unfortunately, surgeons are not so sociable. Strangely, they have become less sociable in hospitals too. There used to be a concept of weekly grand rounds in some hospitals which were attended by all the hospital consultants across the various specialties without fail. The present generation of medicos is unfortunate for being unable to witness such wonderful arguments and counter arguments put forth by eminent medical personnel full of rhetorical and sometimes undiluted humor. Medical college auditoria were a witness to such enlightening and mind-rejuvenating sessions but sadly not anymore. Economic considerations in the private sector have led to absolute rivalry and turf fights. Congeniality and camaraderie is all but forgotten and has been amplified with the rising dissatisfactions of this profession. Isolation and loneliness puts doctors at greater risk of burnout as pointed out in one of the recent articles in the Times of India. They are becoming more irritable, more time-pressured, and less empathic with patients. We should work on this aspect of personal doctor to doctor relationship and improve their interpersonal relationships and their ability at effective communication with co-physicians. My dear friends, it is about time to revive old traditions.

Few days back, a senior surgeon was murdered in Allahabad in his chamber by unknown assailants. 
Similar assaults on doctors are becoming common now. It needs some serious and thorough thinking and action, as we stand united with IMA and other bodies to press our demands and get justice from central and state governments. In spite of Doctors Protection Act, its poor implementation and execution has not given any respite to doctors and a central law to protect medical practitioners and their establishments is the need of the hour.

More than one lakh surgeons are working in the country, yet only about 20,000 are members of ASI. Many surgeons in your neighborhood, in your city, your friends, and your resident colleagues back from college days are attending activities by city surgical bodies. One ASI member encouraging enrollment of his fellow surgeon into ASI would make our strength double fulfilling my dream for ASI for it to touch a membership of 40,000 .

Social Security Scheme for the benefit of members has been revived and details of which are available on the official ASI Website. Professional Indemnity Scheme is also in the process.
I wish to see ASI members as part of a family, to enjoy and participate actively in academics, and to learn and give better surgical care to patients. It needs mutual trust and most importantly belief in self to solve all the problems within the house as per provision of ASI constitution.

Our leaders in the past have laid down a solid platform for this glorious association of immense fame by ornamenting it with all facilities and a bright vision to guide us for the welfare of members in the future. Our responsibility is to preserve its sanctity and its integrity and move forward with each other's help. I have great faith in our system, and the fellow members and time are on our side to create a better future to be a proud Indian surgeon. Future generation of surgeons has many hopes and expectations from us, and we have to perform to prove it.

Long live ASI. 